Instructions for authors, subscriptions and further details:

http://brac.hipatiapress.com

\title{
Metaverso y artista
}

David Serra Navarro ${ }^{1}$

1) Departamento de Filología y Comunicación. Universitat de Girona, España.

Date of publication: October 3rd, 2013

To cite this article: Serra Navarro, D. (2013). Metaverso y artista. BRAC Barcelona Research Art Creation, 1(2), 130-152. doi: 10.4471/brac.2013.06

To link this article: http://dx.doi.org/104471/brac.2013.06

\section{PLEASE SCROLL DOWN FOR ARTICLE}

The terms and conditions of use are related to the Open Journal System and to Creative Commons Non-Commercial and Non-Derivative License. 
BRAC - Barcelona Research Art Creation. Vol. 1 No. 2, October 2013

pp. $130-152$

\section{Metaverse and Artist}

David Serra Navarro

Universitat de Girona

\section{Abstract}

This paper shows an interpretation of the virtual world Second Life through their artistic actions, especially based on the code performance notion, questioning and reflecting on the medium itself. A cross view to make visible the main focus of interest of the metaverse in an approximation of aesthetic analysis, and understanding the creative dispersion that seemingly aimless transits and is manifested in a virtual body.

Keywords: Virtual body, Second Life, relational aesthetics, virtual object, code performance 
BRAC - Barcelona Research Art Creation. Vol. 1 No. 2, October 2013

pp. $130-152$

\section{Metaverso y Artista}

David Serra Navarro

Universitat de Girona

\section{Resumen}

El siguiente artículo muestra una interpretación del mundo virtual de Second Life a través de sus acciones artísticas, especialmente desde la noción code performance, cuestionando y reflexionando sobre el propio medio. Una visión transversal que quiere hacer visible los principales focos de interés de dicho metaverso en una aproximación de análisis estético, y entender su dispersión creativa que transita aparentemente sin rumbo y que se manifiesta en un cuerpo virtual.

Palabras claves: Cuerpo virtual, Second Life, estética relacional, objeto virtual, code performance 
L Los mundos virtuales constituyen un nuevo foco de atención para los creadores de contenidos, pero también suponen un nuevo medio a explorar desde su vertiente artística y sus posibilidades expresivas. La diversidad y complejidad de estas plataformas que configuran los entornos virtuales en línea nos ofrecen un amplio abanico de peculiaridades, estableciéndose como nexo común las características de corporeidad, interactividad y persistencia. Nuestro interés, por este medio transversal y el individuo que lo habita, lo delimitaremos sobre el metaverso ${ }^{1}$ de Second Life. Linden Lab es la corporación responsable de esta propuesta, mostrada al público en el año 2003 bajo la dirección de Philip Rosedale y el determinante asesoramiento de Jaron Lanier y Lawrence Lessig, Second Life promueve una política de contenidos en la que sus usuarios tienen el control de la propiedad intelectual de sus obras, hecho más que significativo para explicar una estimulación creativa en la producción de objetos virtuales. En consonancia, este mundo virtual que funciona con divisas reales, se nutre económicamente del tránsito y consumo virtual, siguiendo un modelo meritocrático en el que sus usuarios también ven recompensadas sus contribuciones. De esta manera, sumergirnos en su posible esfera artística implicará conocer la disolución del «objeto artístico» en un sistema de intercambios y transacciones simbólicas, pero a su vez integrado como un elemento de mercado y lenguaje.

Si bien podemos categorizar la actividad artística del metaverso en función del producto resultante, acercándonos a términos como el hyperformalism de Dan Coyote ${ }^{2}$, la práctica machinima ${ }^{3}$ o la code performance de Gazira Babeli ${ }^{4}$, se sugiere una visión alternativa que pueda dibujarnos un escenario de esta realidad artística que fluye entre la estética, la ética y la política del entorno virtual en cuestión.

\section{La imagen-cuerpo}

Abordar la imagen que discurre en tiempo real $^{5}$ significa entender el objeto virtual como una extensión del cuerpo, y una negociación del sujeto entre lo virtual y su significado. En este sentido, en el proceso de 
transferencia de cuerpo a «objeto-cuerpo», nos lleva a interpretar la figura del artista bajo dos posicionamientos vinculados a la estética relacional de Nicolas Bourriaud. Primeramente como un «semionauta» que navega entre signos y con los que crea nuevas conexiones, pero también como un signo más de este universo. Por lo tanto, este enfoque quiere ser una muestra de apertura conceptual, un planteamiento en el que no se discrimine la producción virtual en función de su apariencia, sino que interconecte la deriva cultural de Second Life a través de subjetividades virtuales y lenguaje. Como nos señala Mike Featherstone (2007), el tramado cultural de imágenes y signos saturan la sociedad, y hacen de la vida un consumo estético que rompen los límites entre arte y vida cotidiana, dándose así una reestructuración de la producción y fuerzas productivas que sitúan las relaciones sociales en el mismo plano de lo económico. En paralelo, siguiendo las argumentaciones de Roy Frankenberger (2008), en que nos describe nuevos modelos de gobernabilidad a raíz de un cambio de poder dominado por la economía de signos y un sentido estético globalizado, nos trasladamos de nuevo sobre la gestión individualizada de la producción de sentido; en este caso retomando el contexto social en el que se inician los procesos de significado-contenido, en una constante revisión del «yo-avatar» en relación al entorno. Así pues, el siguiente artículo pretende reflexionar la interacción en un entorno virtual singular mediante la praxis artística, tomando la performance como eje central en el que el sujeto cuestiona su nuevo cuerpo como instrumento de comunicación y de expresión artística.

\section{Code performance y acción mimética}

Paradójicamente, en un mundo sin restricciones físicas, la copia de lo real marca un itinerario visual en el imaginario de Second Life; ya sea en sus paisajes que clonan grandes ciudades, en sus habitantes bípedos, en la negación de la ingravidez o en sus productos humanizados. La retroalimentación entre ambos mundos se extiende mediante la imagen, conduciéndonos a unas relaciones sociales mediatizadas por la imagensignificado, y que posiblemente nos reflejen la noción de espectáculo de Guy Debord (1967). La simulación parece llenar el inventario de cada avatar, y su producción nos refleja parte de la experiencia depositada en 


\section{David Serra Navarro - Metaverso y artista}

cada usuario, adoptando formas, objetos y prótesis más propias del mundo real que del metaverso. No obstante, en esta manipulación colectiva en la que impera la imagen de lo real, ¿hay cabida para nuevos lenguajes?

Una de las prácticas artísticas más determinantes en la resolución de este interrogante se encuentra en la noción code performance; tal acción consiste en trasladar en Second Life la reproducción real de la acción del cuerpo, a modo de happening, ahora mediante un alter-ego que se inscribe en un entorno virtual. Aparentemente esta es otra modalidad de mímesis, talmente como un performer virtual en el que su nuevo cuerpo se manifiesta como soporte y material artístico, y que nos apunta hacia un catalizador de la condición humana en la perspectiva social y simbólica, su fenomenología existencial y el concepto de biopoder de Foucault (Scheper-Hughes 1994, p. 232). No obstante, en esta concepción del cuerpo como elemento que fluctúa en el espacio y el tiempo, y lugar en donde construir una identidad en relación al otro, no podemos comprender la performance desde la fisicalidad y el dolor a que puede verse sometido el cuerpo; se pierde su dimensión material y se actúa en la descorporeidad. Qué sentido adquieren en el plano virtual las acciones de los artistas Chris Burden, Marina Abramovic o Stelarc?

Tomemos por ejemplo la performer Sigalit Landau en Barbed Hula ${ }^{6}$ (2000), cuyas cicatrices nos remiten a la supresión del ritual y al deseo de ser liberado de las restricciones sociales; mientras, imaginemos su avatar homólogo, el cual sólo recreará el deseo de acercarse a la realidad. En la misma línea, sin riesgo físico, el salto al vacío de Yves Klein (Le Saut dans la Vide, 1960) se desnaturaliza en otro mensaje; ahora el individuo ingrávido está condenado a no lastimarse, a persistir en un bucle sin otro fin que la desconexión de la red. Como nos señala Claudia Giannetti (2003), introduciéndonos en otro concepto paralelo:

El público se transforma en voyeur de su propia metaformance, a la vez que se disipa la división clara entre el cuerpo remoto o telepresente y el cuerpo físico real, ya que el interactor pasa a existir (virtualmente) en y entre ambos lugares. (p.216) 


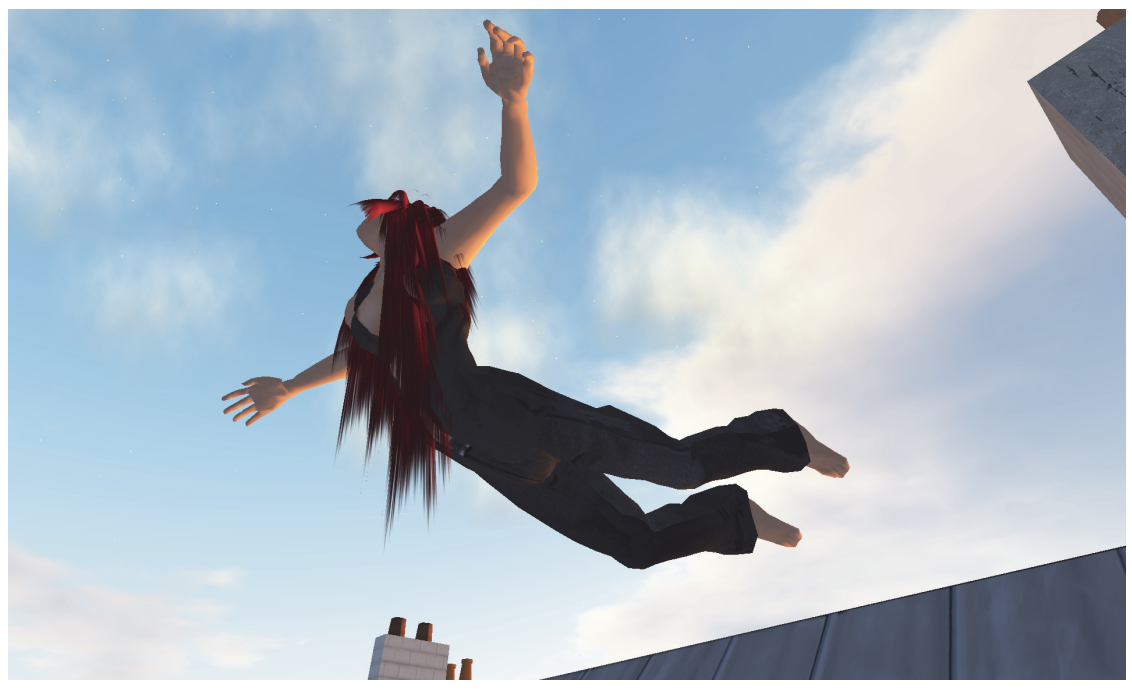

Figure 1. Yves Klein tribute, Kenneth Russo 2010. Esta captura de interfaz corresponde al proyecto Culdesac Island del artista Kenneth Russo, concretamente la imagen muestra un usuario utilizando el código abierto que replicaba la acción de Yves Klein, reviviendo una experiencia inalcanzable e ironizando sobre la condición del avatar. Fuente: Kenneth Russo.

La producción code performance del universo de Linden Lab puede suscitarnos una deriva cultural de creaciones dispersas y efímeras, sin embargo, como figura icónica de esta praxis, cabe citar la obra iniciada en el año 2006 de la artista virtual Gazira Babeli. Sus aportaciones no residen en clonar la historia de la performance, sino en cuestionar el propio medio y el propio cuerpo virtual. Babeli añade una significación a los límites del lenguaje corporal virtual, juega con la plasticidad de la identidad, y reinventa al artista en un marco aún por explorar. Entre sus obras más determinantes encontramos Avatar on Canvas (2007), Come Together (2007) o Ursonate in Second Life - Monument to Kurt Schwitters (2007); un conjunto de propuestas que se muestran al espectador tanto en formato código como en formato objeto, interpelando la experiencia de éste y convirtiéndole en parte del medio, en un «espectactor» (Boal, 1980). Según Domenico Quaranta (2010, p. 
160-175), Gazira Babeli es en ella misma un proyecto artístico, una identidad en un universo simulado, y en esencia el resultado de la manipulación y subversión de los códigos. De esta manera, sumándose a este modo de experimentación virtual popularizado por Babeli, nos encontraremos en paralelo con otros artistas y colectivos que ven en Second Life una alternativa para desenvolver nuevas experiencias: China Tracy con su obra i.Mirror (2007) para la 52 ${ }^{\mathrm{a}}$ Bienal de Venecia, en la que se documentan desde la idea belleza los excesos de consumo y capitalismo virtual; Eva y Franco Mattes con sus Synthetic Performances (2007) reviviendo a Joseph Beuys o Vito Acconci en el metaverso; o incluso el propio Stelarc con Avatars have no organs (2009), una performance que integrará realidad y virtualidad para describirnos un nuevo estadio del cuerpo sin subordinaciones biológicas y una amplificación de la experiencia.

\section{Devenir objeto en movimiento}

En el momento en que el usuario accede a desdoblarse en un nuevo ente virtual, frecuentemente bípedo y con rasgos importados de la realidad, se está produciendo un contrato simbólico y cultural en el que se rompe la dicotomía sujeto-tecnología; un producto final que se materializa en la interfaz, un acto de comunión entre aquello virtual y aquello real. Como nos señala Zygmunt Bauman (2003), en una sociedad "líquida", individualizada y simultáneamente interconectada, se nos aparece constantemente un duelo entre la libertad y la comunidad. La tensión entre lo colectivo y lo personal, nos presenta y representa un juego de procesos de socialización dentro de un imaginario colectivo vivido paradójicamente en el protagonismo individual. En esta colisión de lo virtual y de lo real se inicia la intersubjetivización del individuo, convertido en parte del flujo de información que transita modificando los sentidos del propio medio. Cabe señalar que la intersubjetivización puede considerarse como una extensión de la subjetivización (Traugott, 2003, p. 134), un mecanismo de reclutamiento de significados y que en el este caso el "nuevo individuo" adoptará como herramienta comunicativa. En otras palabras, el sujeto controla su experiencia desde un nuevo ángulo de la realidad, y su vehículo es el cuerpo virtual: un 
objeto de significación cambiante. Del face-to-face al object-to-object, un nueva forma de comunicación vinculada a un diseño de entorno virtual, en este caso el mundo de Second Life.

Por lo tanto, la figura del artista performativo bajo esta óptica nos sugiere una posición de reencuentro, de "participación mágica" (Maffesoli, 2009), que replantea el presente y lo cotidiano en el "movimiento" solitario y compartido. Recordemos a Susanne K. Langer, en su libro Feeling and Form (1953), abordaba la noción de las artes visuales como espacios virtuales en los que: "Una imagen no es en realidad más que un "objeto" exclusivamente virtual. Su importancia radica en el hecho de que no la utilizamos para que nos guíe hacia algo tangible y práctico, sino que la tratamos como una entidad completa que sólo tiene relaciones y atributos virtuales, nada más; todo lo que es, está en su forma visible» (1953, p. 48). Ahora la imagen somos nosotros, tomamos su presencia como un estado de consciencia que nos ayudará a construir nuevos modelos de realidad a través de la participación. Una "imagen-objeto-en movimiento" que supera desde la interactividad la separación cuerpo-mente, y que nos muestra una reestructuración de nuestra percepción y pensamiento, acogiéndose en un nuevo estado del cuerpo como instrumento de intercambio.

El metaverso proporciona la hipermediación y la hibridización de la información, es el paso de la metáfora a la metonimia que disipa lo teatral para entrar en un juego común marcado por la acción del individuo. Esta idea, en que lo dinámico fija un posicionamiento vital, nos aproxima a un amplio marco de trabajo en el que lo imaginario adquiere un valor añadido de realidad; la hipermediación entendida como la inmersión del sujeto en los métodos interactivos de escritura y su relación en distintos grados de significación. Ya no se delimita mediante la representación, el objeto-cuerpo nos permite movernos y sentirnos dentro de aquello intersticial, artístico. En el "analogon" de Second life parece que todo sucede en la pantalla, así cabe diferenciar varios niveles de interacción y una gran variedad de objetos que configuran este entorno; la gradación empieza por el objeto estático persistente y termina en el objeto-cuerpo, dejando entre los dos extremos prótesis, dispensadores, algoritmos con funcionamiento autónomo, o prototipos de inteligencia artificial que derivan por el espacio. Sin embargo, delante esta clasificación objetual no podemos 
olvidarnos de su componente social en el que de forma inmersiva se escenifican una serie de prácticas rituales, vertebrando un juego que une multiplicidades de experiencias y que crea sentido de comunidad. Comprender el nuevo yo significa descubrir el propio entorno, y esta realidad conducida mediante lo performativo como vía de liberación se desenvuelve en un contexto liminal. Victor Tuner nos describía:

«...liminality represents the midpoint of transition in a statussequence between two positions, outsiderhood refers to actions and relationships which do not flow from a recognized social status but originate outside it, while lowermost status refers to the lowest rung in a system of social stratification in which unequal rewards are accorded to functionality differentiated positions» (1974, p. 237)

De esta manera, Second Life se puede entender como una interfaz asociada al espacio liminal, un canal de transición en el que se potencia la experiencia del cuerpo. Una estrategia de construcción de experiencia común, en la dispersión y en la fluidez; talmente como una fusión del teatro y lo ritual que nos acerca a la idea de Jerzy Grotowski de comunicación espiritual, una ceremonia que lleva a los "espectadores" a la catarsis mediante el trabajo (acción) del actor. En esta línea, José Antonio Sánchez nos apunta:

El cuerpo subjetivo mantiene una potencialidad movilizadora que incrementa su eficacia en sociedades marcadas por la hipermediación y la teatralidad generalizada. La movilización puede producirse como acción, situación, recorrido o encuentro $\mathrm{y}$ contribuir desde la co-presencia a pensar nuevos modos de relación (2009, p. 8)

El vínculo del cuerpo como objeto de negociación nos transmite una escena compartida de consumo de experiencias, una unidad social que impulsivamente utiliza el movimiento para desarrollar su identidad. 


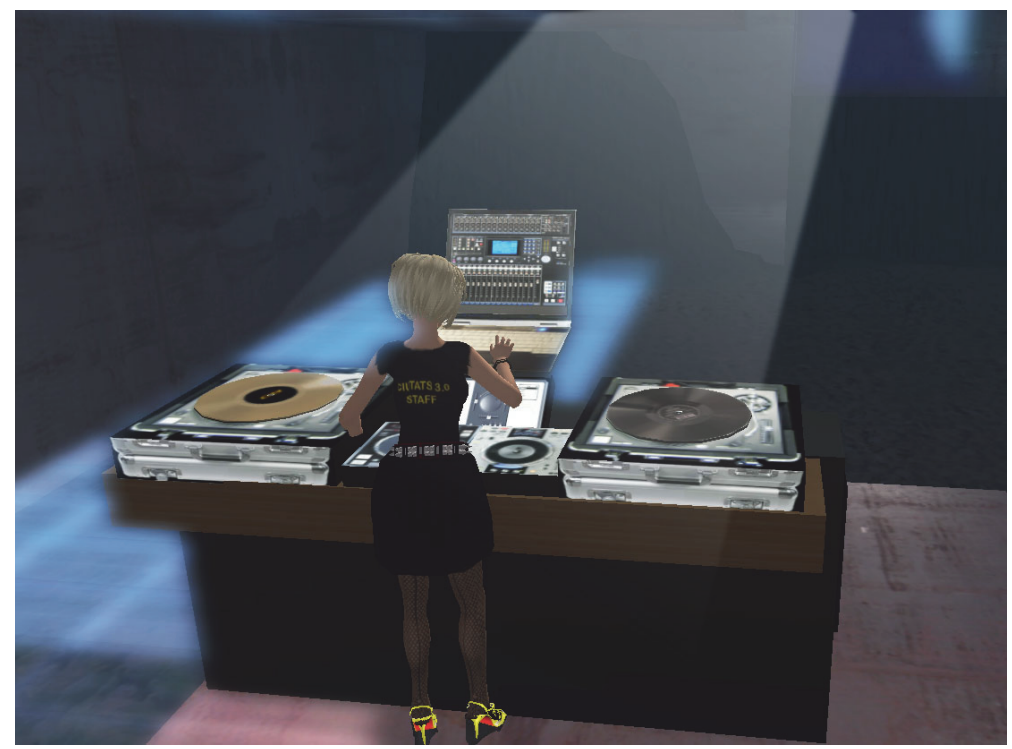

Figure 2. Avatar DJ en sala virtual. Posiblemente la escenificación de las raves sea una de las manifestaciones más gráficas en que cultura, cuerpo e identidad se funden en modo ritual. Estas acciones de baile frenético suelen reproducirse en el marco de Second Life, tomando de nuevo el cuerpo como protagonista. Fuente: Kenneth Russo.

\section{Ecosistema económico y contributivo}

A diferencia de los populares servicios de redes sociales, la plataforma de Philip Rosedale, Second Life, no basa su negocio interno en la acumulación de usuarios, apropiación de contenidos e impacto publicitario. Su política defiende la propiedad intelectual de cada residente virtual, o dicho de otra manera, el trabajo puede convertirse en recompensa. Si bien el incentivo más visible es el económico, también se contemplan unos valores meritocráticos hacia la contribución de conocimiento. Un ejemplo que simplificaría esta lógica pudiera ser la creación de un "objeto-prótesis" que pese a un valor económico nulo (freebie) se difundiera entre la comunidad, ya sea como expresión estética compartida (moda), o reconocimiento hacia su autor. 


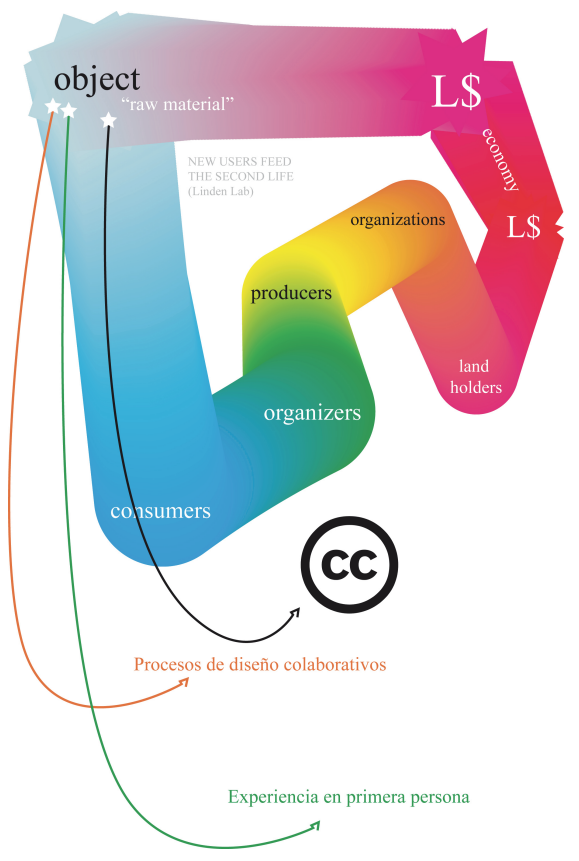

Figure 3. Esquema de retroalimentación económica del medio. El objeto virtual es una pieza clave en el engranaje de negocio, pero también es la herramienta que confiere actividad creativa al usuario. Fuente: autor.

Bajo estas directrices, basadas en motivar la creación de objetos, se nos plantean varias visiones sobre el tipo de producción individual: el objeto como mercancía, el objeto como anexo al cuerpo y/o el objeto como delimitador del espacio. ¿Dónde situar el cuerpo? Quizás las posibilidades funcionales de los objetos vayan más allá de esta simplificación clasificatoria, no obstante se nos dibuja un panorama en el que la imagen es la mediadora comunicativa y el organismo avatar le proyecta velocidad, tiempo real. Las creaciones se acumulan en todos los rincones del metaverso, en los supermercados automatizados, en los inventarios de los avatares o inmortalizadas en las instantáneas de los usuarios; en definitiva son el alfabeto que les permite articular significado. Esta realidad, y no ficción, desencadena un conjunto de 
procesos relacionales en los que el cuerpo no se queda al margen. Lo performativo es el motor que pone en circulación lo creado, unos valores comunicativos que impregnan cada cuerpo de forma cambiante, popular y sin otra restricción que la libertad de elección de su portador. De nuevo libertad y comunidad son los dos ejes que sacuden al alter-ego en un continuum que debe gran parte de sus sinergias al diseño del medio, una singularidad en la red. Por lo tanto el artista de la acción se acaba mezclando con la acción de la comunidad, y sus contribuciones se hibridizan entre códigos, objetos e imaginario colectivo.

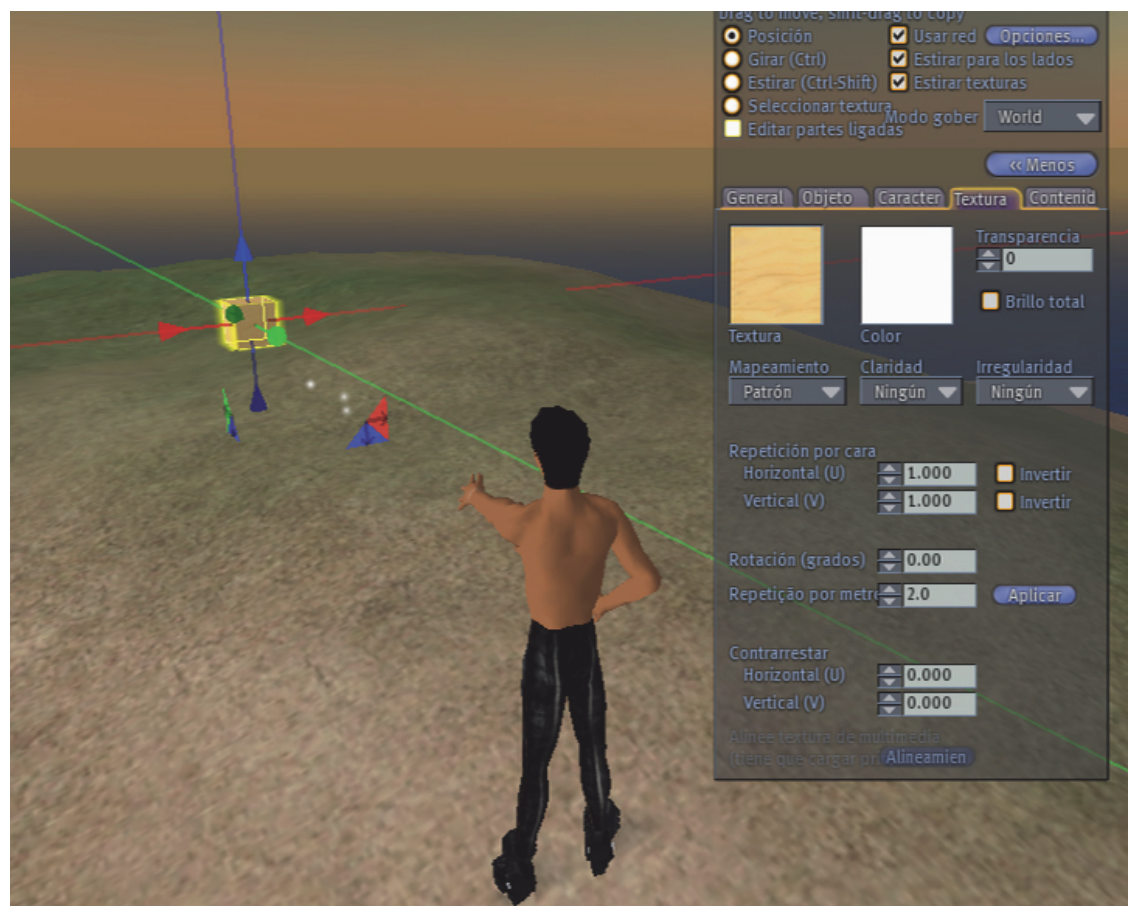

Figure 4. Avatar creando objeto en un sandbox. Second Life proporciona de forma abierta un gestor-modelador 3D de contenidos, en el que los residentes acceden de manera intuitiva, y que no precisa grandes conocimientos de programación LSL (Linden Script Language). El sistema también ofrece la posibilidad de acceder a múltiples bibliotecas y tutoriales de programación más compleja. Fuente: autor. 


\section{El cuerpo contenedor}

No sería del todo objetivo decir que en Second Life existe libertad para crear en cualquier terreno, sólo los propietarios previo pago pueden depositar y exhibir sus objetos. Esto significa que se crea una limitación de posibilidades hacia los usuarios que no disponen de suficientes recursos económicos para comprar suelo virtual con dinero real. Sin embargo, en esta jerarquía que repercute en la modelación del paisaje, encontramos unas zonas llamadas arenales (sandboxes) que cumplen una función muy específica, permitir el desarrollo creativo y el testeo algorítmico bajo una limitación de tiempo. Es decir, son unos espacios de experimentación abiertos, pensados para promover el talento y la actividad productiva, pero con una persistencia de pocas horas. Se puede crear pero no se puede exponer, con lo que probablemente el "producto" nunca será encontrado durante el transcurso de una exploración del lugar. Son objetos efímeros. La única manera que se contempla para salvaguardar este material es trasladarlo dentro del inventario (disco duro interno del registrado) o anexarlo a una parte del cuerpo, utilizando la superficie de la anatomía como una prolongación del suelo virtual. Precisamente en esta última peculiaridad, que inicialmente responde más a una cuestión técnico-empresarial de cómo gestionar y optimizar los privilegios de un objeto, nos encontramos con un nuevo soporte expositor: el cuerpo.

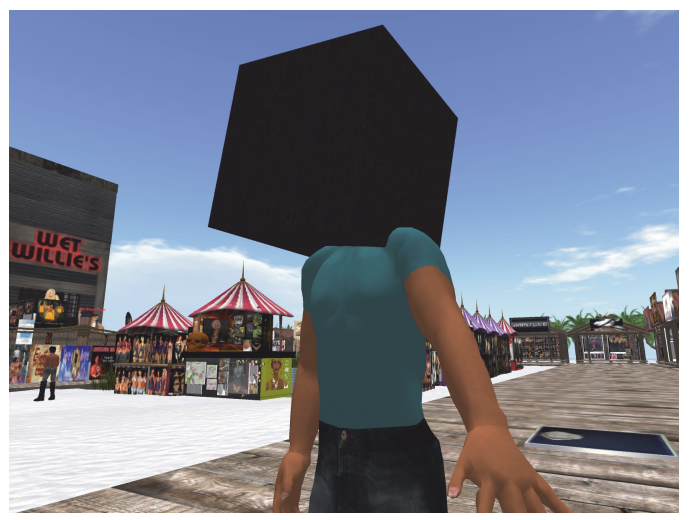

Figure 5. Objeto anexado en cabeza. Los objetos pueden fusionarse en cualquiera de las partes anatómicas del avatar. Estos polígonos base (prims) modifican la apariencia del usuario y establecen nuevas formas de comunicación visual. Fuente: autor. 
Cada avatar utiliza su representación para desplazarse, pero también como portador de los objetos virtuales cuyos autores no poseen privilegios de owner (generalmente en manos de otras corporaciones). La cuestión es que más allá del terreno virtual el cuerpo amplifica su capacidad estrictamente de representación, ya que nos sugiere un propio entorno de investigación semiótica en el que indagar los límites de nuestra proyección. En este sentido, Douglas Rushkoff nos señala: «Our digital experiences are out-of-body. This biases us toward depersonalized behavior in an environment where one's identity can be a liability» (2010, p. 79). Por esta razón, el foco de estas metaestructuras de lenguaje convergen en un yo abstracto, en formación y rizomático; un contorno pixelado que no puede detenerse y que se cuestiona a sí mismo en su línea de tiempo. En consonancia, retomando la obra de Gazira Babeli Come Together, una performance en que los cuerpos virtuales de los espectadores se fusionaban con el propio performer, nos delata este estado cambiante; un cuerpo que nos fuerza a interrogarnos sobre nuevas relaciones entre el sujeto y el mundo en su sentido más amplio. El artista conoce el poder de la acción, pero su "obra" se nutrirá de la interrelación con los procesos metacognitivos de los usuarios que le rodean; en este caso usuarios que aprehenden de, y con, su cuerpoavatar.

\section{Participación}

Poniendo en duda la libertad de los usuarios, desde una perspectiva de la estética normalizada del metaverso, se nos aparecen una serie de acciones subversivas que "combaten" las normas del propio medio. Son actos de resistencia, de manifestación, de contestación social, y que responden al uso del nuevo cuerpo como instrumento de agitación virtual. Muestras de resistencia simbólica que raramente se pueden trasladar al mundo real, y que por esta misma razón dotan de sentido el vínculo avatar-entorno. Frecuentemente, y tomando de referencia el pionero grupo de performers Second Front ${ }^{6}$ (2006), nos encontramos con diferentes estrategias comunicacionales basadas en el mensaje irónico y en la representación metafórica. Tal como nos define la declaración de artista de Liz Solo, miembro del colectivo Second Front, 
sus influencias provienen de: «...numerous sources, including Dada, Fluxus, Futurist Syntesi, the Situationist International and contemporary performance artists such as Laurie Anderson and Guillermo GomezPena, Second Front creates score-based performances and interventions that challenge notions of traditional performance, virtual embodiment and the culture of immateriality». ${ }^{7}$

Un dato que nos lleva a interpretar el metaverso más allá del tráfico de información, que nos induce a interrelacionar consciencias cambiantes que hablan a través de su cuerpo, de un mundo corporal y relacional. Un cuerpo que se opone a un metaverso construido a base de mezclas de fragmentos independientes de realidad, y que unifica relacionalmente la superficie que simula en el entorno. Lo subversivo es visualizar los procesos de producción cognitiva, la pantalla es el cuerpo. Este fenómeno está directamente unido a las nuevas formas sociales de consumo digital, transformando los papeles del espectador $\mathrm{y}$ despertando nuevas audiencias que quieren formar parte de la propia acción. Como nos apuntaba Sara Diamond, las nuevas tecnologías nos piden otra forma de diálogo con el mundo:

«..technology is a material force: while social, cultural and economic structures shape technology, technology also acts back on social, cultural, economic and physical bodies. The materiality of technology and of the image (which acts not only on language but as a language on us) are in constant tension..» (Diamond, 1996, p. 134).

Una producción cultural que se encadena con formas de consumo identidario (Featherstone, 2007), y que afirma al propio sujeto en la individualidad. La performance consumida, desde el cuerpo y en el cuerpo ("exocuerpo digital"), nos construye narraciones en la interconsciencia de un inevitable ser humano que vive y persiste, pero que también convive en la acción. Se trata de la creación de un marco sociocultural articulado, un espacio de creación de formas culturales y un consumo de experiencias que el mundo material no puede satisfacer. Cada avatar determina su destino en un mundo igualitario sin desventajas socioeconómicas (Kelly, 2004, p. 62-63). La disolución de 
las fronteras, en un encuadre ubicuo y simultáneamente autónomo en las experiencias en primera persona, que toman lo performativo como propagador de nuevas realidades. Una forma de arte que se extiende en modo de metacognición, que refleja y se refleja en una realidad rizomática de procesos vitales. En esta sintonía, rescatamos la figura de Nicolas Bourriaud y su entendimiento relacional:

La globalización es económica. Punto. El arte sólo sigue los contornos de ella, porque es el eco, más o menos lejano, de los procesos de producción [...] el arte no procede por imitación de procedimientos y modos contemporáneos, sino según un juego complejo de resonancias y resistencias que a veces lo acerca a la realidad concreta, otras lo aleja hacia formas abstractas, o arcaicas. [...] El arte da cuenta de la evolución de los procesos productivos en su globalidad, de las contradicciones entre las prácticas, de las tensiones entre la imagen que una época crea de sí misma y la que proyecta realmente. En una época en que las representaciones se interponen entra la gente y su vida cotidiana, o entre los seres humanos mismos, es absolutamente normal que el arte se aleje a veces de la representación para transformarse en una parte de la realidad en sí. (Bourriaud, 2009, p, 177)

La estética relacional concibe la realidad material de la forma como relacional, ya que la experiencia formal de la relación produce realidad; es decir, según Bourriaud no hay existencia real sin la interacción, que es la que promueve las formas democráticas de participación artística. Dejando atrás la contemplación y la función de almacenamiento cultural, esta teoría relacional nos acerca a un campo más amplio, en el que el arte está incluido pero que se despliega la forma globalmente. En esta línea José Luís Brea nos señala: «...la energía simbólica que moviliza la cultura está empezando a dejar de tener un carácter primordialmente rememorante, recuperador, para derivarse a una dirección productiva, relacional. [...] Para en cambio convertirse en esa herramienta mediante la que inteligimos el mundo colectivamente.» (Brea, 2007, p. 5). 
Esta visión del mundo articulado culturalmente entra en sincronía con la definición de Félix Guattari y Gilles Deleuze de la obra de arte como bloques de perceptos y de afectos, que revisada en clave actual encuentra en la complejidad de las nuevas tecnologías un nuevo tipo de forma-mundo. En la convergencia arte-ciencia-tecnología se nos presenta el concepto espacio-tiempo como lugar practicable. Tiempo interactivo, tiempo del perpetuum mobile, que interferirá en la imagen en el tiempo de anunciación de esta; aquello virtual subvertirá el registro tradicional del tiempo, y el espacio será el resultado de esta actualización constante. Al trasladarnos en el metaverso, esta reflexión estética no se producirá sobre la forma instrumental o representativa, sino sobre las transformaciones e hibridaciones en la poética de aquello tecnológico. Un proceso a través de la imagen digital interactiva confiere sentido a los objetos. Por lo tanto, el cuerpo es el portador de la acción, interactiva, instantánea, y que dará pie a un sistema activo de captación y evolución de la información. El cuerpo como un fluir en el medio, y que cuestiona al spect-actor mediante prácticas perfomativas las dicotomías natural-artificial o naturaleza-cultura, estableciéndose como un lenguaje propio que modela nuevos modelos de comunicación no verbal, que implica a otros cuerpos, y que en su vertiente de fuerza comunicante construye nuevos códigos. Otra particularidad del cuerpoavatar es que el usuario reviste su apariencia para mostrarse al mundo pero también para definirse en el mundo, talmente como un voyeur de su propia representación. Así pues, se actúa a partir de una imagen del signo que se desearía ser, olvidando el espejo de Lacan en que el usuario se representaría creyendo que es. Una muestra de transitoriedad, de movimiento, y comportamiento social, que en ojos de Gilles Lipovetsky podría interpretarse como "la moda que teje sociedad":

«We have reached the era of consummate fashion, the extension of the fashion process to broader and broader spheres of collective life. Fashion is not so much a particular peripheral sector, now, as a general form at work in society as a whole. Everyone is more or less immersed in fashion, more or less everywhere.» (Lipovetsky, 1994, p. 131). 


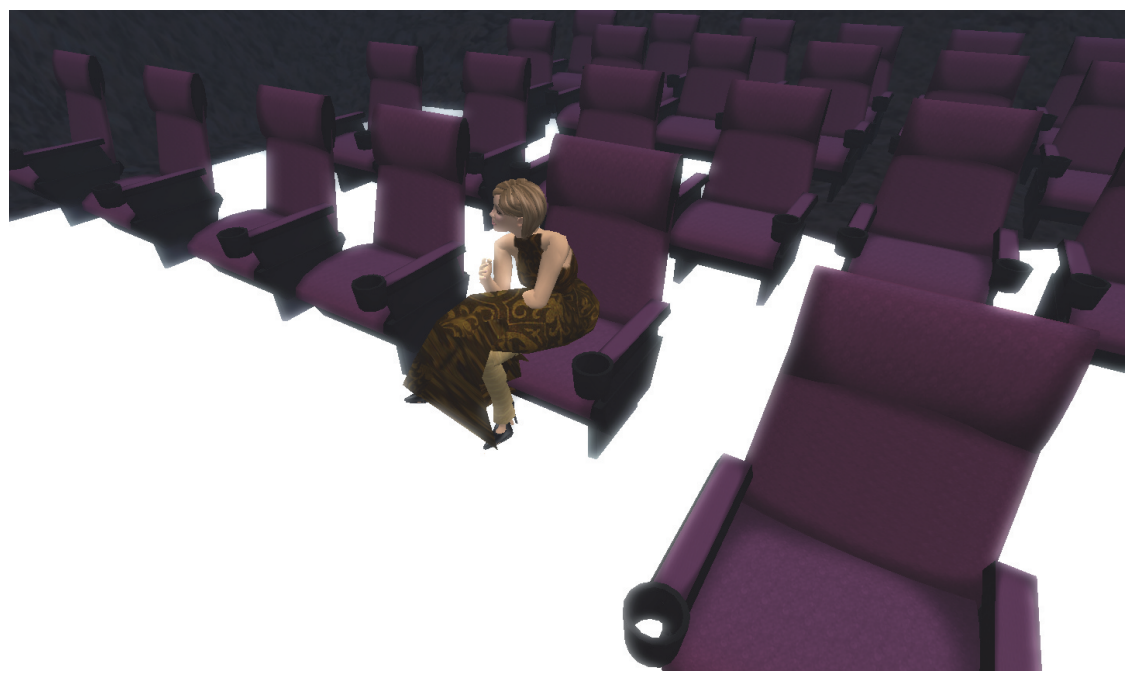

Figure 6. Avatar en sala de proyección vacía. Los espacios del metaverso establecen una relación de intersubjetividad con el usuario, no obstante es en la relación concreta cuerpo-espacio donde se interpela al usuario. Fuente: autor.

Lo participativo, el encaje y diálogo de los cuerpos, fundamenta la acción artística y las negociaciones de sentidos. En la acción deviene un intercambio simbólico, otra experiencia, que como nos describe Rosanne Stone: «Penetrating the screen involves a state change from the physical, biological space of the embodied viewer to the symbolic, metaphorical consensual hallucination of cyberspace; a space that is a locus of intense desire for refigured embodiment.» (Stone, 2000, p. 522). El metaverso nos proporciona otro contexto para expandir nuestro cuerpo, y salvando las distancias la performance nos remite a una estética interactiva que no siendo material se abraza a la idea duchampiana de vida, o al sentido vital de las composiciones de John Cage, tomando al "otro" que se escapa del simulacro. De forma concisa, Diana Domingues nos apunta:

El ciberarte, por la interactividad, demanda la presencia del cuerpo en acción. Sus interacciones generan ambientes vivos. Así, es una 
forma de arte que estrecha sus relaciones con la vida, reforzando la premisa duchampiana de aproximar el arte a la vida. En las interacciones no es más el objeto que interesa, sino un campo de relación que se realiza en estados imprevisibles, de regeneraciones y que ocurre entre el cuerpo y las tecnologías, en una "zona de intervalo" donde algo acontece y que no podría existir sin la presencia y acción del cuerpo con las tecnologías con las cuales está interactuando.» (Domingues, 2000, p. 7).

Interacción y sistema son nodos claves de un proceso abierto de cocreación de experiencias en un medio transmedia, induciendo a producir nuevos relatos en comunidad y desde el cuerpo-nodo. La cuestión es que nuestra "presencia" adquiere un nuevo envolvente dentro esta caja escénica que es Second Life, una ilusión que sólo puede llevarnos al significado comprendiendo el estado intersticial del medio; la acción se diluye en códigos, y la figura del performer se nos aparece en la pantalla en luz-objeto que confiere pequeños fragmentos de realidad a "otros" interactuantes-luz. Nos situamos en un punto medio, en una zona de contacto, en un cuerpo-interfaz que reclama su camino errático mediante el movimiento. La inmanencia propia de las experiencias tintadas de lo tecnológico nos destapan un nuevo conocimiento, y un nuevo modelo vida para los sujetos; un giro radical que Scott Lash nos describirá de la siguiente manera: "Culture is displaced into an immanent plane of actors attached or interfaced with machines. Now we experience cultural things not as trascendental representations, but instead as immanent things: as objects, as technologies» (Lash, 2002, p. 9).

\section{Comunicación y objeto}

El código, el que posibilita nuestro alter virtual, es una herramienta de subversión que encarna un poder simbólico a través de su representación objetual. Nuestro avatar no sólo representa, sino que presenta un modo de actuación, estableciéndose una negociación entre nuestra identidad en formación y el objeto virtual que lo esculpe ante 
nuestros ojos. La batalla de estas aproximaciones artísticas no tiene lugar en el cuerpo individual fenomenológico, su atractivo se perfila como un proceso fusión entre lenguaje y límites relacionales; una convergencia entre el objeto semiótico y la subjetividad virtual de quién decide controlarlo. Por esta razón, sin categorizar, y sin ahondar en la dispersa especificidad de la producción virtual, intuimos como el medio Second Life deviene un espacio-tiempo intersticial en el que desarrollarse artísticamente. Más allá de una comunidad en red, el metaverso se articula como un laboratorio social en el que sus usuarios utilizarán su nuevo cuerpo-objeto para comunicarse, interactuar y descubrir conexiones entre ambos mundos. Sin ánimo de quitar importancia a los nuevos ismos que surgen en este entorno, ya que demuestran un amplio repertorio de expresiones y fragmentos significantes, en su análisis global, el signo en tiempo real nos delata otra visión del escenario artístico más cohesionado y más ubicuo. Con esta visión de pájaro, y dejando atrás muchos otros elementos no circunstanciales, como el valor económico del signo, se nos despliega un océano a medio camino entre lo real y lo virtual; un espacio para explorar en primera persona y vivirlo en clave real, ya sea definiendo una extensión de nuestro cuerpo, comprendiendo nuevas relaciones con el mundo o preguntándonos si realmente la contribución del artista en Second Life es difundir conocimiento. Todo desde la acción, el cuerpoacción del metaverso.

Quizás el salto al vacío no ha hecho más que empezar, hacia un futuro incierto en que se diluyen nuevas formas relacionales de entender y habitar el ciberespacio estéticamente.

\section{Notas}

${ }^{1}$ El término metaverso se identifica con la idea de universo paralelo, y proviene de la novela Snow Crash de Neal Stephenson (1992); también se utiliza habitualmente para describir la visión del trabajo en espacios 3D totalmente inmersivos.

2 Véase trabajos de Dan Coyote en línea: http://www.dancoyote.com/ Recuperado el 9 de mayo de 2011.

${ }^{3}$ El machinima, contracción de los términos machine y cinema, hace referencia a un género audiovisual que utiliza generalmente los gráficos de los videojuegos como elementos de una narración. Se constituye como un espacio donde confluye cine, 
animación y juego; una animación que es capturada en tiempo real en un entorno virtual.

4 Véase trabajos de Gazira Babeli en línea: http://www.gazirababeli.com/WORKS.php Recuperado el 10 de junio de 2010.

5 Hay que precisar que la idea de tiempo real en Second Life está condicionada por el tiempo de reacción de sus servidores, siendo en muchos casos más una intención que una realidad. Véase los términos asociados: rezzing $i$ derezzing en: http://secondlife.wikia.com/wiki/Rez Recuperado 10 de julio de 2012.

6 Véase video en línea: http://www.videoartworld.com/video_66.html Recuperado 14 de julio de 2011.

7 Véase integrantes del grupo (entre ellos Gazira Babeli) y documentación en línea de sus performances en: http://www.secondfront.org/index.html Recuperado el 4 de febrero de 2012.

${ }^{8}$ Véase Artist's Statement en página web personal de Liz Solo:

http://www.lizsolo.com/secondfront.html Recuperado el 7 de febrero de 2012.

\section{Referencias}

Bauman, Z. (2003). Comunidad. En busca de seguridad en un mundo hostil. Madrid: Siglo XXI.

Boal, A. (1980). Stop: c'est magique! Río de Janeiro: Civilização Brasileira.

Bourriaud, N. (2006). Estética relacional. Buenos Aires: Adriana Hidalgo.

Bourriaud, N. (2009). Radicante. Buenos Aires: Adriana Hidalgo.

Brea, J. L. (2007). Cultura RAM, Mutaciones de la cultura en la era de su distribución electrónica. Barcelona: Gedisa.

Debord, G. (1967). La société du spectacle. Paris: Les Éditions Gallimard (1992).

Diamond, S. (1996). Technology matters: material issues in the information age. En: Peter White (ed) Naming a Practice, Curatorial Strategies for the Future (Banff: Walter Phillips Gallery Editions, 1996).

Domingues, D. (2000). Cibercultura, creación e interactividad. En:

FERLA, Jorge La. (Org.). De la pantalla al arte transgénico.

Buenos Aires: Livros del Rojas - Universidad Buenos Aires.

Featherstone, M. (2007). Consumer Culture and Postmodernism. 2nd

Edition. London, Thousand Oaks, New Delhi: SAGE

Publications.

Frankenberger, R. (2008, 25 de junio). Learning from Baudrillard and 
Foucault: Consumer Culture, Social Milieus and the Governmentality of Lifestyle, $31^{\mathrm{a}}$ conferencia anual ISPP, París. Giannetti, Cl. (2003). Arte humano/máquina: virtualización, interactividad y control. En: Hernández Sánchez, D. Arte, cuerpo, tecnología. Ediciones Universidad Salamanca, p. 216.

Graham, E. L. (2002). Representations of the Post-Human: Monsters, Aliens, and Others in Popular Culture. New Brunswick, NJ: Rutgers University Press.

Kelly 2, R.V., (2004). Massively Multiplayer Online Role-Playing Games. North Carolina, USA: McFarland \& Company, Inc. Langer, S. K. (1953). Feeling and Form: A Theory of Art. New York: Charles Scribner's.

Lanier, J. (2010). You are not a gadget: A manifesto. New York: Alfred A. Knopf.

Lash, S. (2002). Critique of Information. London: Sage.

Lessig, L. (2004). Free Culture: How Big Media Uses Technology and the Law to Lock Down Culture and Control Creativity.

Recuperado el 3 de mayo de 2008, de www.jus.uio.no/sisu/free_culture.lawrence_lessig/portrait.pdf Lipovetsky, G. (1994). The Empire of Fashion: Dressing Modern Democracy. Trad. Catherine Porter. Princeton: Princeton Univ. Press.

Maffesoli, M. (2009). El reencantamiento del mundo. Una ética de nuestro tiempo. Buenos aires: Dedales.

Quaranta, D. (2010). Media, New Media, Postmedia. Milano: Postmediabooks.

Rushkoff, D. (2010). Program or Be Programmed. Ten Commands for a Digital Age. New York: OR Books.

Sánchez, J. A. (2009). Teatralidad y Cultura Visual. Revista 0000. Museo Reina Sofía: Madrid, pp. 8-11.

Scheper-Hughes, N. (1994). Embodied Knowledge: Thinking with the Body in Critical Medical Anthropology. En: Assessing Cultural Anthropology. Rob Borofsky, ed. New York: McGraw-Hill. Serra, D. (2012). Culdesac Island [194/204/21]. Girona: Documenta Universitaria.

Stone, R. A. (2000). En: Bell, D.; Kennedy, B.: The cybercultures 
152 David Serra Navarro - Metaverso y artista

reader. London: Routledge.

Traugott, E. C. (2003). From subjectification to intersubjectification. En:

Raymond Hickey (ed.), Motives for Language Change. Cambridge:

Cambridge University Press, pp. 124-139.

Turner, V. (1974). Dramas, Fields, and Metaphors: Symbolic Action in

Human Society. Ithaca, NY: Cornell University Press.

David Serra Navarro es Profesor Visitante de la Universitat de Girona, Departamento de Filología y Comunicación UdG. España.

Contact Address: Universitat de Girona. Departamento de Filología y Comunicación. ST. DOMÈNEC. Plaza Ferrater Mora 1. 17071 - GIRONA. España. E-mail address:

david.serranavarro@udg.edu 